\title{
Swarmic Sketches and Attention Mechanism
}

\author{
Mohammad Majid al-Rifaie ${ }^{1}$ and John Mark Bishop ${ }^{2}$ \\ 1 Vividus Solutions LTD., Vividus Swarm Lab, London EC1V 2NX \\ m.majid@vividusolutions.com \\ ${ }^{2}$ Goldsmiths, University of London, New Cross, London SE14 6NW \\ m.bishop@gold.ac.uk
}

\begin{abstract}
This paper introduces a novel approach deploying the mechanism of 'attention' by adapting a swarm intelligence algorithm - Stochastic Diffusion Search - to selectively attend to detailed areas of a digital canvas. Once the attention of the swarm is drawn to a certain line within the canvas, the capability of another swarm intelligence algorithm - Particle Swarm Intelligence - is used to produce a 'swarmic sketch' of the attended line. The swarms move throughout the digital canvas in an attempt to satisfy their dynamic roles - attention to areas with more details - associated to them via their fitness function. Having associated the rendering process with the concepts of attention, the performance of the participating swarms creates a unique, non-identical sketch each time the 'artist' swarms embark on interpreting the input line drawings. The detailed investigation of the 'creativity' of such systems have been explored in our previous work; nonetheless, this papers provides a brief account of the 'computational creativity' of the work through two prerequisites of creativity within the swarm intelligence's two infamous phases of exploration and exploitation; these phases are described herein through the attention and tracing mechanisms respectively.
\end{abstract}

Keywords: Stochastic Diffusion Search, Particle swarm optimisation, sketching, drawing, attention.

\section{Introduction}

Studies of the behaviour of social insects (e.g. ants and bees) and social animals (e.g. birds and fish) have proposed several new metaheuristics for use in collective intelligence. Natural examples of swarm intelligence that exhibit a form of social interaction are fish schooling, birds flocking, ant colonies in nesting and foraging, bacterial growth, animal herding, brood sorting etc.

Following other works in the field of swarms painting (6]16/20|21]) and ant colony paintings (1015), the outputs presented in this paper - created by a swarm intelligence algorithm - are also used as a platform to argue whether or not swarm intelligence algorithms have the potential to exhibit computationally creativity.

Although producing artistic works through the use of swarm intelligence techniques have been previously explored, this work explores the concepts of attention and creativity through this type of collective intelligence, which emerges 
through the interaction of simple agents (representing the social insects and animals) in nature-inspired algorithms - Stochastic Diffusion Search (SDS) [7] and Particle Swarm Optimisation (PSO) [13].

In this work, SDS is deployed to enforce the idea of attention to area of the search space (digital canvas with line drawings) where there are more details (i.e. more lines); once the area of attention is identified, PSO through its particles, traces the points of the lines selected and its particles' movement are visualised on the canvas. As attention moves from one area of the original line drawing to another, a sketch is produced which is the collective result of the SDS-led attention and PSO-led tracing mechanism.

In the last couple of years, there has been several research work utilising the two aforementioned swarm intelligence algorithms; while scientific merits of integrating these algorithms are investigated in detailed (e.g. [4), their artistic capabilities have been detailed in several publications along with some philosophical arguments on computational creativity (e.g. [1/5|2]).

In the next section (Section 2), the swarm intelligence algorithms used are explained. Subsequently, a historical perspective of attention is presented (Section (3) followed by explanation on the attention and tracing mechanisms associated with SDS and PSO algorithms respectively, providing details on the performance of the computer-generated nature-inspired attentive swarms in re-interpreting the original line drawings (Section 4). Finally a conclusion and summary of the work are given.

\section{Swarm Intelligence}

This section describes two nature-inspired swarm intelligence algorithms: Stochastic Diffusion Search - inspired by a species of ants and uses communication as its main mean to converge to an optimum food location by recruiting individual ants - and Particle Swarm Optimisation - simulating the choreography of fish schooling or birds flying.

\subsection{Stochastic Diffusion Search}

This section introduces Stochastic Diffusion Search (SDS) 7] - a swarm intelligence algorithm - whose performance is based on simple interaction of agents. This algorithm is inspired by one species of ants, Leptothorax acervorum, where a 'tandem calling' mechanism (one-to-one communication) is used, the forager ant that finds the food location recruits a single ant upon its return to the nest; therefore the location of the food is physically publicised [14].

The SDS algorithm commences a search or optimisation by initialising its population and then iterating through two phases (see Algorithm 1)

In the test phase, SDS checks whether the agent hypothesis is successful or not by performing a hypothesis evaluation which returns a boolean value. Later in the iteration, contingent on the precise recruitment strategy employed (in the diffusion phase), successful hypotheses diffuse across the population and 


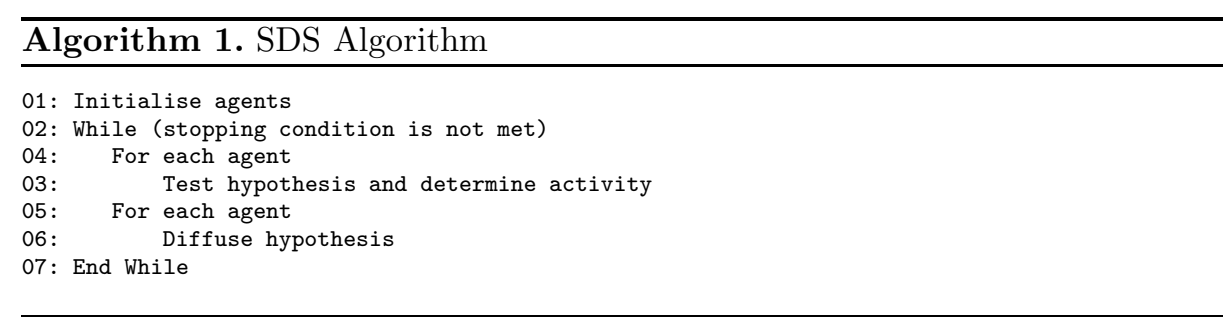

in this way information on potentially good solutions spreads throughout the entire population of agents. In other words, each agent recruits another agent for interaction and potential communication of hypothesis.

In standard SDS (which is used in this paper), passive recruitment mode is employed. In this mode, if the agent is inactive, a second agent is randomly selected for diffusion; if the second agent is active, its hypothesis is communicated (diffused) to the inactive one. Otherwise there is no flow of information between agents; instead a completely new hypothesis is generated for the first inactive agent at random. Therefore, recruitment is not the responsibility of the active agents.

\subsection{Particle Swarm Optimisation}

A swarm in Particle Swarm Optimisation (PSO) algorithm comprises of a number of particles and each particle represents a point in a multi-dimensional problem space. Particles in the swarm explore the problem space searching for the optimal position, which is defined by a fitness function.

Each particle has a position $x$, a velocity $v$, and a memory, $p$, containing the best position found so far during the course of the optimisation, and this is called the personal best (pbest). $p$ can also be thought of as a particle 'informer'. Particles participate in a social information sharing network. Each particle is informed by its neighbours within this network, and in particular, the best position so far found in the neighbourhood, is termed the neighbourhood best. The position of each particle is dependent on the particle's own experience and those of its neighbours.

The standard PSO algorithm defines the position of each particle by adding a velocity to the current position. Here is the equation for updating the velocity of each particle:

$$
\begin{gathered}
v_{i d}^{t}=w v_{i d}^{t-1}+c_{1} r_{1}\left(p_{i d}-x_{i d}^{t-1}\right)+c_{2} r_{2}\left(g_{i d}-x_{i d}^{t-1}\right) \\
x_{i d}^{t}=v_{i d}^{t}+x_{i d}^{t-1}
\end{gathered}
$$

where $w$ is the inertia weight whose optimal value is problem dependent [17; $\boldsymbol{v}_{i d}^{t-1}$ is the velocity component of particle $i$ in dimension $d$ at time step $t-1 ; c_{1,2}$ 
are the learning factors (also referred to as acceleration constants) for personal best and neighbourhood best respectively (they are constant); $r_{1,2}$ are random numbers adding stochasticity to the algorithm and they are drawn from a uniform distribution on the unit interval $U(0,1) ; p_{i d}$ is the personal best position of particle $x_{i}$ in dimension $d$; and $g_{i d}$ is the neighbourhood best. Therefore, PSO optimisation is based on particles' individual experience and their social interaction with other particles. After position and velocity updates, the positions of the particles are evaluated and the memories $p$ are updated, if a better position has been found.

In this paper, Clerc and Kennedy's PSO (PSO-CK [9]) or constriction PSO is used:

$$
v_{i d}^{t}=\chi\left(v_{i d}^{t-1}+c_{1}^{\prime} r_{1}\left(p_{i d}-x_{i d}^{t-1}\right)+c_{2}^{\prime} r_{2}\left(g_{i d}-x_{i d}^{t-1}\right)\right)
$$

where $\chi=0.72984$ [ , which is reported to be working well in general, is used in this work.

\section{Attention}

For centuries, attention has been preoccupying the minds of philosophers and psychologists and scientists. The concept of attention has been studied mostly in psychology and neuroscience (see Table 1.1 in Phuong Vu: Historical Overview of Research on Attention, in 22 for more details) and there has been considerably less notable interest on attention within the field of computational creativity.

In the early $18^{\text {th }}$ century attention was mostly seen as a way of abstraction (see Berkeley's 1710 theory of abstract ideas in [18]):

"[It] must be acknowledged that a man may consider a figure merely as triangular, without attending to the particular qualities of the angles or relations of the sides. So far he may abstract, but this will never prove that he can frame an abstract general, inconsistent idea of a triangle."

By 1769, when Henry Home Kames added the appendix of 'Terms Defined or Explained' to his Elements of Criticism [12, attention's role as a regulator of cognitive input was regarded as definitive of it:

"Attention is that state of mind which prepares one to receive impressions. According to the degree of attention objects make a strong or weak impression. Attention is requisite even to the simple act of seeing."

Thus, regulating cognitive and sensory inputs was associated to attention. Later, William James in The Principles of Psychology in 1890 [1] offered a more comprehensive description of attention (i.e. focalisation, etc.):

"Every one knows what attention is. It is the taking possession by the mind, in clear and vivid form, of one out of what seem several simultaneously possible objects or trains of thought. Focalization, concentration, of consciousness are of its essence [...]" (p. 403-404) 
and few pages further, he continues:

"Each of us literally chooses, by his ways of attention to things, what sort of a universe he shall appear to himself to inhabit." (p. 424)

Two decades later, in 1908, as emphasised by E.B. Titchener [19], attention was given a greater significance :

"What I mean by the 'discovery' of attention is the explicit formulation of the problem: the recognition of its separate status and fundamental importance; the realization that the doctrine of attention is the nerve of the whole psychological system, and that as men judge of it, so they shall be judged before the general tribunal of psychology."

and its importance grew over the years in psychology and neuroscience. Although the concept of attention might have been present in the work of some researchers in the field of computational creativity, the focus on attention has not been equally considerable among researchers in this field; perhaps, partly because there has not been a clear definition on attention.

The next section adopts a particular type of attention (i.e. attention to detailed regions of the canvas) and expands on its application in the context of sketching swarms (or swarmic sketches).

\section{Attention and Creativity in the Swarms}

In this section, the attention mechanism, which is controlled by SDS algorithm is detailed; this is followed by the process through which PSO algorithm utilises the output of the SDS-led attention to visualise the particles movements on the digital canvas which produces the final sketch rendered by the swarms.

\subsection{Attention Mechanism}

The input digital image consists of lines (see Fig. 1) each formed up of a series of points (the image on the left is after one of Matisses sketches).

The swarms' attention in this work is controlled by the level of the intensity in the drawings within a specific radius, $r a$ of an agent. In other words, the intensity or fitness of an agent, $f_{i,(x, y)}$, where $i$ is the agent number and $(x, y)$ is the coordinate of the agent in the search space (input image), is calculated by the number of points constituting the drawing within the radius ra. (see Fig. 2a)

As mentioned earlier in Section 2.1. each agent has two components: status, which is a boolean value and hypothesis. The hypothesis of each agent in this work is the $(x, y)$ coordinate which is used to calculate the fitness, $f_{i,(x, y)}$, of the agents located at any particular pixel within the input image.

After the agents are randomly initialised throughout the search space (Fig. 3a), in order to determine the status of an agent, $i$, within the swarm (test 

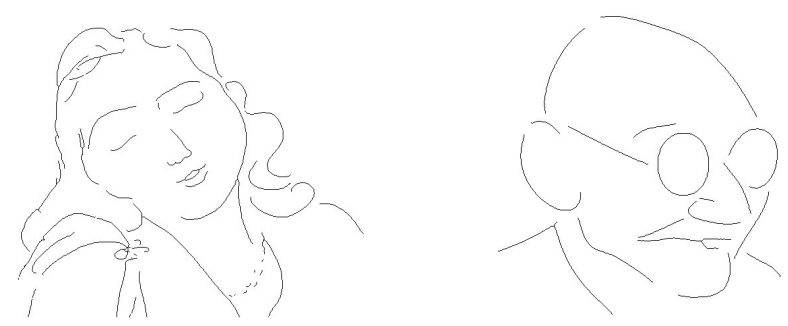

Fig. 1. Input images: series of points forming line drawings

phase), its fitness, $f_{i}$, is calculated as explained above and another agent, $r$, is randomly selected; if $f_{i}>f_{r}$ (i.e. the agent $i$ is located in a more line intense area), agent $i$ is set active, otherwise inactive (Fig. 3b illustrates active agents in red and inactive ones in black).

In the diffusion phase, as in standard SDS, each inactive agent randomly pick another one. If the randomly selected agent is active, the inactive agent adopts the hypothesis of the active one. However, if the selected agent is inactive, the selecting agent generates a random hypothesis $(x, y)$ from the search space. See Fig. 3r for the behaviour of the agents after the diffusion phase; the area with the best fitness (most line intense area) is highlighted with a circle.

After $n$ number of test-diffusion phases cycles, the biggest cluster of the agents is identified and the closest point $\left(p_{c}\right)$ to the cluster is calculated. Once the $(x, y)$ coordinate of the point is retrieved, the starting and end points of the line is extracted and a string of $(x, y)$ coordinates from starting to end points of the line is passed on to the PSO particles to trace one by one. Fig. 20 shows the identified ends of a selected line.

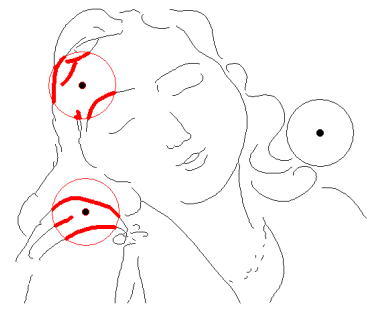

(a)

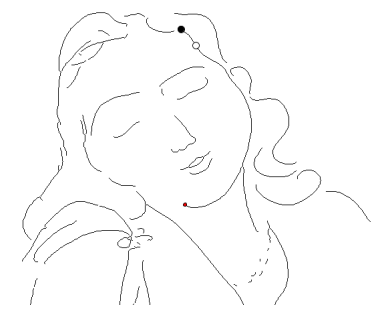

(b)

Fig. 2. (a) Agent's fitness: in this figure, the $(x, y)$ coordinates of three exemplar agents are illustrated with black dots in the centre of the circles; the highlighted points of the line drawing within each circle contribute towards the fitness of the agent, $f_{i,(x, y)}$. (b) Selected line: The hollow circle represents the selected point, $p_{c}$ and the two ends of the line - start and end - where $p_{c}$ resides, are highlighted in black and red, respectively. 


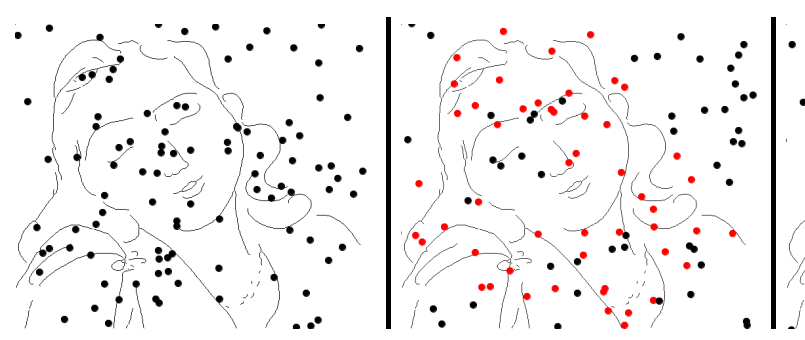

(a) (b)

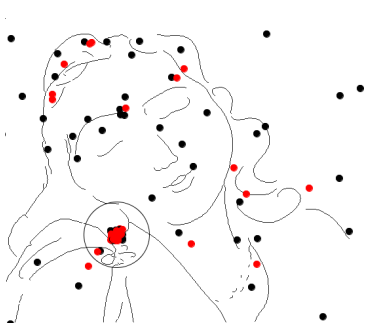

(c)

Fig. 3. SDS stages: (a) Initialisation; (b) Test Phase; (c) Diffusion

\subsection{Tracing Mechanism}

The points creating the lines of the line drawing are treated as targets by the PSO algorithm. Thus, particles aim to trace these points one at a time until reaching the end of the line (the algorithm tries to minimise the distance between each particle's position and the point it aim to track).

Particle's movement is visualised on the canvas (i.e. trajectory of the particles moving from position $\left(x_{0}, y_{0}\right)$ to $\left(x_{1}, y_{1}\right)$ and so forth). The adopted PSO algorithm is briefly presented in Section 2.2 (more technical details on the behaviour of particles are reported in a previous publication [1]).

As stated earlier, input to PSO algorithm is a series of points forming up a line, which among other points, consists of the starting and end points, as well as $p_{c}$. The algorithm is then instructed to trace the line commencing from $p_{c}$ to the beginning of the line, and them back towards the end of the line drawing. Once the line is traced, it is removed from the search space and the other lines are considered one by one according to the attention mechanism deployed.

This process ensures that in addition to the potential aesthetic of the swarms' final sketches, the process of sketching is enriched with attention to details. See Fig. 4 for the final output of the swarm: 1. Fig. 5 shows another output of the introduced mechanism.

In this work $r a$ is set to $50, w=550, h=450$, the population size of SDS and PSO are set to 100 and 10 respectively and $n=20$.

\subsection{Discussion}

Using the analogy of 'first come, first serve', this work biases the attention towards 'more details, first sketch'. The value of $n$, which is the number of testdiffusion cycles before picking a line to trace, controls the precision of attention. The larger the value is, the more precise the attention is (i.e. focusing on the detailed parts of the input image); and the smaller the value, the less accurate the attention would be. Fig. [6 illustrates the process for $n=10$ which allows

\footnotetext{
${ }^{1}$ The HD video recordings of few instances of the performance of the swarms are accessible at http://youtu.be/-VYBi-awPUo or http://youtu.be/BFIJrvcNEFA
} 




Fig. 4. Output sketch of the swarms using both SDS-led attention mechanism and PSO-led tracing mechanism

the SDS agents enough time to converge on the detailed area as the sketching process progresses; on the other hand, Fig. 7 where $n=1$, shows the lack of attention to detailed area of the input image.

While the finaloutput of the swarms is an important part of the process - mainly executed through PSO algorithm - the primary contribution of the attention mechanism - facilitated by SDS algorithm - lies in the dynamic leadership of the sketching process, as it progresses versus the final graphic output solely.

Although this work uses Stochastic Diffusion Search to intelligently (vs. greedily or randomly) control the attention of the swarms (by identifying the detailed regions of the canvas to start the sketching), the concept of attention is extendible to other measures such as colour, shapes, etc., which are currently being explored. See [3] for an example of using SDS-led colour attention for rendering input images into paintings called 'Swarmic Paintings'.

The general behaviour of the swarms in the context of computational creativity has been extensively discussed in previous works ([1/2/5]), touching upon the concepts of freedom and constraint and their impact on mapping these two prerequisites of creativity onto the two well-known phases of exploration and exploitation in swarm intelligence algorithms. Although most swarm intelligence algorithm have their internal exploration and exploitation phases, in this work, 




Fig. 5. A swarmic sketch made from an input image after one of Picasso's sketches Reverdy (Pierre), Cravates de Chanvre

the global exploration of the search space is carried out through the attention mechanism and the exploitation phase is facilitated through the tracing mechanism. 


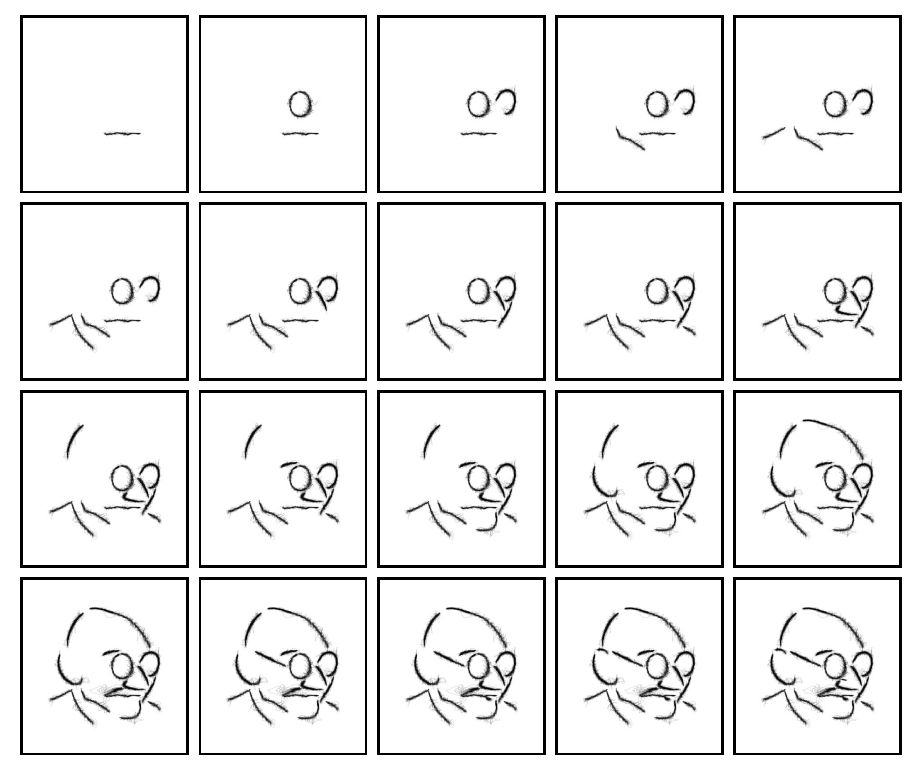

Fig. 6. Output sketch with $n=10$, where the swarms attention is drawn towards sketching the detailed area first

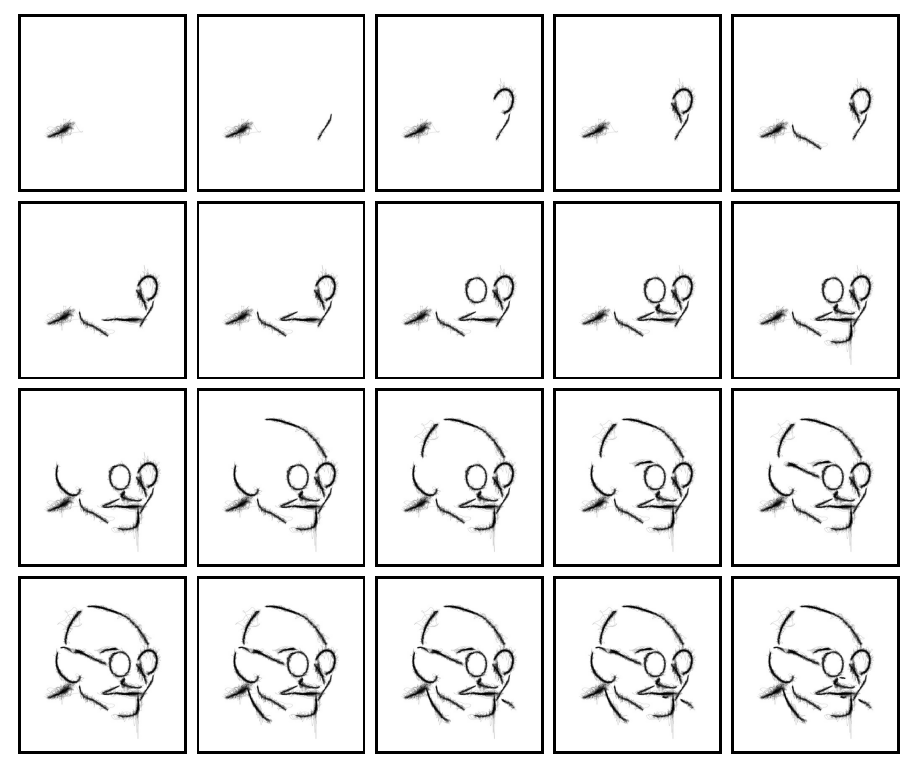

Fig. 7. Output sketch with $n=1$, which results in less accurate attention of the swarms in first picking the detailed area of the line drawing 


\section{Conclusion}

The so described computational artist is the outcome of a novel marriage between two classical swarm intelligence algorithms (Stochastic Diffusion Search and Particle Swarm Optimisation), which deploy 'attention' in the production of 'traced' line drawings. The adapted SDS algorithm utilises the agents with dynamically changing 'attention' through adopting the lines falling in the detailed areas of the input image. The agents thus communicate the details to PSO algorithm, which in turn traces the points of the line with its particles; the movements of the dynamic particles are visualised on the canvas as part of the sketching process of the swarms. This process is repeated for all the lines of the input image, and the outcome - emerging through millions of simple interactions - produces (although loyal, yet) non-identical sketches of the same input line drawings whenever the process is repeated.

This work also highlights the mechanisms responsible for the exploration and exploitation phases within the swarm intelligence algorithms and their relationship with freedom and constraints as two prerequisites of creativity. In brief, expanding on the previous research on computational creativity, in addition to the practical aspect of the work presented, this work introduces the SDS-led attention mechanism utilised for producing sketches from input line drawings. Finally, the concept of SDS-led attention is extendible to other measures (e.g. colour, shapes, etc.) which are currently being investigated.

\section{References}

1. Al-Rifaie, M.M., Aber, A., Bishop, M.: Cooperation of nature and physiologically inspired mechanisms in visualisation. In: Ursyn, A. (ed.) Biologically-Inspired Computing for the Arts: Scientific Data through Graphics. IGI Global, United States (2012) ISBN13: 9781466609426, ISBN10: 1466609427

2. Al-Rifaie, M.M., Bishop, M.: Weak vs. strong computational creativity. In: AISB 2012: Computing and Philosophy, University of Birmingham, Birmingham, U.K. (2012)

3. Al-Rifaie, M.M., Bishop, M.: Swarmic Painting with Colour-attentive Swarms. In: Machado, P., McDermott, J., Carballal, A. (eds.) EvoMUSART 2013. LNCS, vol. 7834, pp. 97-108. Springer, Heidelberg (2013)

4. Al-Rifaie, M.M., Bishop, M., Blackwell, T.: An investigation into the merger of stochastic diffusion search and particle swarm optimisation. In: GECCO 2011: Proceedings of the 2011 GECCO Conference Companion on Genetic and Evolutionary Computation, pp. 37-44. ACM (2011)

5. Al-Rifaie, M.M., Bishop, M., Caines, S.: Creativity and autonomy in swarm intelligence systems. In: Bishop, M., Erden, Y. (eds.) Journal of Cognitive Computation: Computational Creativity, Intelligence and Autonomy, vol. 3, pp. 320-331. Springer (2012)

6. Aupetit, S., Bordeau, V., Monmarche, N., Slimane, M., Venturini, G.: Interactive evolution of ant paintings. In: The 2003 Congress on Evolutionary Computation, CEC 2003, vol. 2, pp. 1376-1383 (2004)

7. Bishop, J.: Stochastic searching networks. In: Proc. 1st IEE Conf. on Artificial Neural Networks, London, UK, pp. 329-331 (1989) 
8. Bratton, D., Kennedy, J.: Defining a standard for particle swarm optimization. In: Proc of the Swarm Intelligence Symposium, pp. 120-127. IEEE, Honolulu (2007)

9. Clerc, M., Kennedy, J.: The particle swarm-explosion, stability, and convergence in amultidimensional complex space. IEEE Transactions on Evolutionary Computation $6(1), 58-73$ (2002)

10. Greenfield, G.: Evolutionary Methods for Ant Colony Paintings. In: Rothlauf, F., Branke, J., Cagnoni, S., Corne, D.W., Drechsler, R., Jin, Y., Machado, P., Marchiori, E., Romero, J., Smith, G.D., Squillero, G. (eds.) EvoWorkshops 2005. LNCS, vol. 3449, pp. 478-487. Springer, Heidelberg (2005)

11. James, W.: The principles of psychology (1890)

12. Kames, H.H.: Elements of Criticism (1769)

13. Kennedy, J., Eberhart, R.C.: Particle swarm optimization. In: Proceedings of the IEEE International Conference on Neural Networks, vol. IV, pp. 1942-1948. IEEE Service Center, Piscataway (1995)

14. Moglich, M., Maschwitz, U., Holldobler, B.: Tandem calling: A new kind of signal in ant communication. Science 186(4168), 1046-1047 (1974)

15. Monmarche, N., Aupetit, S., Bordeau, V., Slimane, M., Venturini, G.: Interactive evolution of ant paintings. In: McKay, B., et al. (eds.) 2003 Congress on Evolutionary Computation, vol. 2, pp. 1376-1383. IEEE Press (2003)

16. Moura, L., Ramos, V.: Swarm paintings-nonhuman art. Architopia Book, Art, Architecture and Science pp. 5-24 (2007)

17. Shi, Y., Eberhart, R.C.: Parameter Selection in Particle Swarm Optimization. In: Porto, V.W., Saravanan, N., Waagen, D., Eiben, A.E. (eds.) EP 1998. LNCS, vol. 1447, pp. 591-600. Springer, Heidelberg (1998)

18. Taylor, C.C.W.: Berkeley's theory of abstract ideas. The Philosophical Quarterly 97-115 (1978)

19. Titchener, E.B.: Lectures on the elementary psychology of feeling and attention. The Macmillan Company (1908)

20. Urbano, P.: Playing in the Pheromone Playground: Experiences in Swarm Painting. In: Rothlauf, F., Branke, J., Cagnoni, S., Corne, D.W., Drechsler, R., Jin, Y., Machado, P., Marchiori, E., Romero, J., Smith, G.D., Squillero, G. (eds.) EvoWorkshops 2005. LNCS, vol. 3449, pp. 527-532. Springer, Heidelberg (2005)

21. Urbano, P.: Consensual Paintings. In: Rothlauf, F., Branke, J., Cagnoni, S., Costa, E., Cotta, C., Drechsler, R., Lutton, E., Machado, P., Moore, J.H., Romero, J., Smith, G.D., Squillero, G., Takagi, H. (eds.) EvoWorkshops 2006. LNCS, vol. 3907, pp. 622-632. Springer, Heidelberg (2006)

22. Vu, P.: Historical Overview of Research on Attention. Sage Publications, Incorporated $(2003)$ 